\title{
Video-Based Tasks for Emotional Processing Rehabilitation in Schizophrenia
}

\author{
R. Caballero-Hernández A. Vila-Forcén S. Fernandez-Gonzalo J.M. Martínez-Moreno M. \\ Turon R. Sánchez-Carrión and E.J. Gómez
}

\begin{abstract}
Schizophrenia is a mental disorder characterized by a breakdown of cognitive processes and by a deficit of typical emotional responses. Effectiveness of computerized task has been demonstrated in the field of cognitive rehabilitation. However, current rehabilitation programs based on virtual environments normally focus on higher cognitive functions, not covering social cognition training. This paper presents a set of video-based tasks specifically designed for the rehabilitation of emotional processing deficits in patients in early stages of schizophrenia or schizoaffective disorders. These tasks are part of the Mental Health program of Guttmann NeuroPersonalTrainer ${ }^{\circledR}$ cognitive tele-rehabilitation platform, and entail innovation both from a clinical and technological perspective in relation with former traditional therapeutic contents.
\end{abstract}

Keywords-NeuroPersonalTrainer ${ }^{\mathbb{R}}$, Schizophrenia, Emotional processing, Video, Tasks.

\section{INTRODUCTION}

Schizophrenia is a severe mental disorder, characterized by profound disruptions in thinking, affecting language, perception, and the sense of self. It often includes psychotic experiences, such as hearing voices or delusions. This mental disorder affects about 7 per thousand of the adult population, mostly in the age group 15-35 years [1].

Deficits in social functioning, including communicating with others, maintaining employment, and functioning in the community, are observed in many disorders but are a defining feature of schizophrenia [2].Social cognition refers to the mental operations underlying social interactions, which include processes involved in perceiving, interpreting, and generating responses to the intentions, dispositions, and behaviors of others [3].

Most of the cognitive research in schizophrenia has focused on the following areas: emotional processing, theory of mind, social perception, social knowledge, and attributional bias. Emotional processing [3] refers broadly to aspects of perceiving and using emotion, and it relies on separate prerequisite abilities that are in the process of being identified.
Cognitive rehabilitation programs aim to restore normal functioning or to compensate cognitive deficits that involve higher cognitive functions (attention, memory, executive functioning and language). Besides pharmacological treatment and behavioral therapy, cognitive rehabilitation has been recognized as an important tool in the treatment of schizophrenia [4].

The combination of cognitive rehabilitation, virtual environments and collaborative tele-rehabilitation platforms is on the side of the efficacy of the treatment and maximizes the opportunity of the nervous system to reorganize those functions that will restore higher functionality in relation to Activities of Daily Living (ADL), improving patients' quality of life [5]. In addition, based on the principle that action observation activated the same neural structures responsible for the actual execution of those actions, several studies [6] have proved that ADL observation treatment using video is a good rehabilitative approach in patients with cognitive disorders.

Nowadays, several tele-rehabilitation platforms offer cognitive rehabilitation programs for schizophrenia patients: RehaCom [7], Gradior [8], PssCogReHab [9], BrainTrain [10], etc. However, available programs focus on main cognitive functions, not covering specific needs on social cognition training.

Guttmann NeuroPersonalTrainer ${ }^{\circledR}$ (NPT) [11][12] is a cognitive tele-rehabilitation system that allows the neuropsychologist to schedule a personalized rehabilitation program, each session consisted of specifically designed tasks, according to the patient's cognitive profile, offering an additional way of communication between therapists and patients. In addition, the platform offers a knowledge management module that allows the optimization of cognitive rehabilitation processes. NPT offers several modules of tasks for different neurological disorders, such as acquired brain injury, dementia, intellectual disability and mental illness, with specific modules for children.

Neuropsychologists from Parc Taulí Mental Health Hospital [13] have designed the Social Cognition Module (SCM, from now onwards), as part of NPT's Mental Health program. The aim of this module is to cover the specific 
needs related with social cognition of patients with schizophrenia or schizoaffective disorders in early stages. SCM is compound of four different units: Emotional Processing, Cognitive Bias, Theory of Mind and Final Unit.

In this paper, we present a set of video-based tasks that compounds the SCM's Emotional Processing Unit, as part of NPT's therapeutic contents.

\section{TASKS IMPLEMENTATION}

This process entails a clinical-technological collaborative work, which results critical for the success of the whole project. Tasks implementation goes through four different phases, described below:

\section{A. Requirements Analysis}

The requirements analysis process entails the following steps: Clinical problem, studying schizophrenia and rehabilitation process to understand the specific needs of this kind of patients; Technical study of NPT platform, how it works and its software architecture; and specifications analysis. Neuropsychologists define the design of each interface element and the behavior of each task through a collaborative environment for tasks specification and validation [14] developed at the Bioengineering and Telemedicine Center (GBT-UPM).

\section{B. Design}

Every task has a common interface design, which aims to achieve the maximum usability, based on the special characteristics of schizophrenia patients. In general, a simple design is desired to make the patient feel in a well-known environment even though exercises change along the treatment. For improving usability, we have worked on patterns of consistency and coherence in order to maintain uniformity in screen design. We take care of the placement of the different elements. Likewise, the same color remains to the common elements of the different tasks for an easier and more intuitive identification. Screenshots of task interface are shown in Fig. 1 as an example.

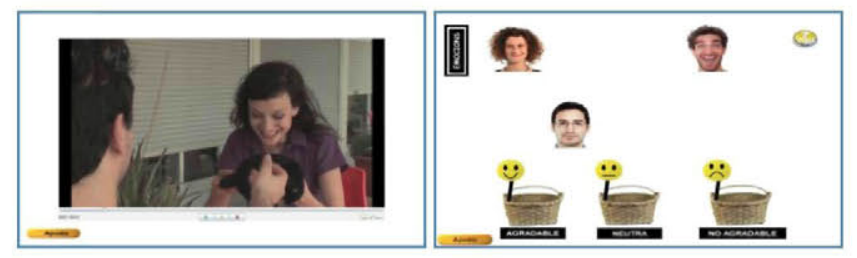

Fig. 1 Examples of Emotional Processing Tasks
As for the execution, all the tasks follow a common sequence of well-defined actions. Tasks behavior can be modeled by a state diagram in which each state represents a screen. The transition between states can be determined automatically or by a user event, such a mouse click, or a temporal trigger. The detailed sequence between the different states is shown in Fig. 2.

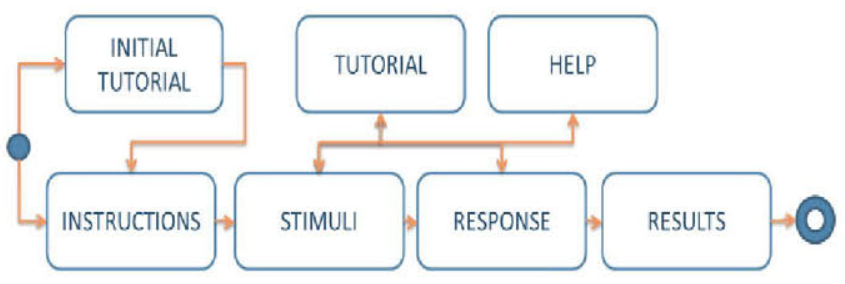

Fig. 2 State Diagram of tasks' execution

\section{- Initial Tutorial}

Shows a test exercise and several tutorial screens if the neuropsychologist configures the task for the tutorial to be shown.

\section{- Instructions}

Shows the directions for completing the task.

\section{- Stimuli}

Generates task stimuli (images, texts, sounds, videos, relationships, etc.) and presents them to the patient for him to interact with the exercise.

\section{- Response}

Gives feedback to the patient about his performance during the exercise.

\section{- Help}

Displays a help message (usually instructions reminder) if the patient requires so.

\section{- Turorial}

Displays the initial tutorial if the patient requires so.

\section{- Results}

Represents patient's outcomes both numerically and graphically.

\section{Software Implementation}

Once the design process is completed, software implementation process begins. Java programming language is used for this. This language is chosen because NPT has been developed in this language, which means a restriction factor for selecting another one.

The programming scheme is based on a parent class, whose properties are inherited by all the classes that consti- 
tute a task. In addition, there is a set of classes that encapsulate a variety of common attributes and functions that are necessary for the development, representing entities such as the display or controlling the communication with the platform for launching the task.

In this work we introduce an innovation in stimuli representation in relation to traditional 2D-tasks [5]: the possibility of video visualization. For this, we use DJ Project, an open source project that allows playing a video in Java using VLC libraries as backend. To keep the integration with the NPT platform, it was necessary to create several classes to interact with the DJ Project library. One of the classes creates a panel and includes a video container while another class controls the video playback inside the task. A task can access to all functions of this new class and play a video when a screen is loaded or when the patient clicks a button, among other actions.

\section{Technical Evaluation}

Once a task is implemented, it is time to verify if it fulfills the initial specifications. As the specification, technical evaluation is carried out through the collaborative environment mentioned before [12], where engineers and neuropsychologists can access to a validation module (specific for each task) in which every aspect that must be checked is represented. A color code is used to indicate developers if a field has been checked with a positive or negative result (green/red), or to indicate neuropsychologists that it is waiting for validation (orange), acting like some kind of semaphore. This is a critical phase where a lot of information is exchanged between developers and neuropsychologists.

\section{EMOTIONAL PROCESSING UNIT}

As a result of this work, a set of 15 computerized tasks has been obtained. These tasks compound the NPT's Emotional Processing Unit, which is organized in blocks, as shown in Table 1.

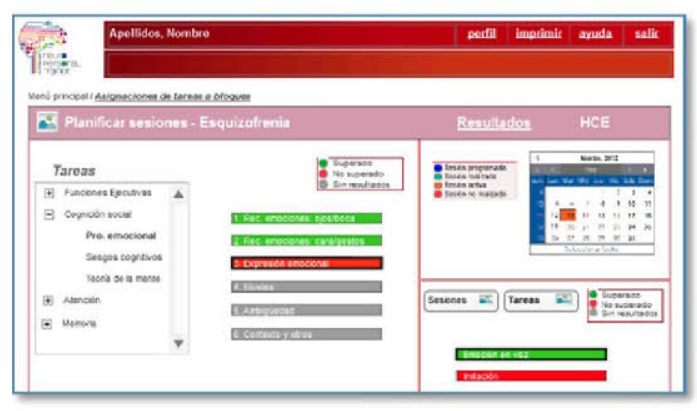

Fig. 3 NPT scheduler
Table 1 Emotional Processing Module

\begin{tabular}{|c|c|}
\hline Block & Task \\
\hline \multirow{4}{*}{$\begin{array}{l}\text { 1. Emotion Recognition } \\
\text { (Eyes/mouth) }\end{array}$} & Eyes \& Mouths \\
\hline & Discover the photograph \\
\hline & Face puzzle I \\
\hline & Same emotion \\
\hline \multirow{4}{*}{$\begin{array}{l}\text { 2. Emotion Recognition } \\
\text { (Face/gesture/emotion/ } \\
\text { voice type) }\end{array}$} & Faces \& Expressions \\
\hline & Pleasant vs. Unpleasant \\
\hline & The box \\
\hline & Voice emotion \\
\hline \multirow{2}{*}{ 3. Emotion Levels } & Order the emotions \\
\hline & Face puzzle II \\
\hline \multirow{2}{*}{ 4. Ambiguity } & Ask for more information I \\
\hline & Ask for more information II \\
\hline \multirow{3}{*}{ 5. Context \& Others } & End of the story \\
\hline & How will the character respond? \\
\hline & What does the character feel? \\
\hline
\end{tabular}

Tasks in each block shares the same therapeutic goal:

\section{Emotion Recognition (Eyes/mouth)}

To recognize basic emotions (happiness, sadness, anger, surprise, etc.) based on eyes and mouth expression.

\section{Emotion Recognition (Face/gesture/emotion/voice type)}

To recognize basic and more complex emotions combining face expression with postural signs and gestures. Also, to identify whether an emotion is positive, negative or neutral.

\section{Emotion Levels}

To identify both the type and degree (little, enough, and much) of an emotion, based on abilities trained in previous modules.

\section{Ambiguity}

To be able to analyze and interpret an ambiguous situation, asking for more information when necessary.

\section{Context \& Others}

To work out how does a character feel or how he will react based on a particular context, previously presented.

Each block represents a more difficult challenge for the patient to achieve. Therefore, neuropsychologists have determined that planning tasks from one block must be restricted by the accomplishment of the previous ones. Consequently, NPT scheduler has been modified for SCM so 
that therapists can check whether the patient has completed a block or not by a color legend, as shown in Fig. 3 .

Since May 2012, a randomized clinical trial (RCT) has been conducted at Parc Tauli Mental Healt Hospital [13] to test the effectiveness of this therapeutic program. Patients in the experimental group perform sessions of computerized tasks from SCM, planned by therapists, up to approximately 40 hours of treatment. Patients in the control group perform nonspecific office automation tasks and video viewing sessions for the same hours. Every subject is neuropsychologically assessed before and after the intervention, as well as three months after the end of it.

Preliminary results of 32 patients show an improvement in problem resolution, verbal memory, selective attention and emotional processing in the experimental group compared to the control one after the treatment. In spite of the small sample size of the RCT, results yielded by now suggest that SCM has a positive impact in the rehabilitation of patients in early stages of schizophrenia or schizoaffective disorder.

\section{DISCUSSION ANDCONCLUSIONS}

This paper presents a set of tasks that compound NPT's Emotional Processing unit.

From a technological perspective, video technology has been incorporated to the traditional 2D-tasks. For this, tasks framework has been modified and extended, providing a series of new classes and methods which open the door to the incorporation of another technologies in the near future, such as flash animations or interactive video [15].

Regarding economic aspects, it is obvious that video production is expensive. Professional actors have participated in the recording, which has been carried out by a film producer. Therefore, a video bank has been created so that clips can be reused in future tasks, resulting in a reduction in the development costs.

From a clinical point of view, this module focus on social cognition deficits, which remained uncovered by cognitive tele-rehabilitation programs up to now. Therefore, schizophrenia patients, who typically present this kind of disorders, can receive a complete rehabilitation program through NPT, combining both cognitive (attention, memory and executive functioning) and social cognitive tasks in their rehabilitation sessions. Preliminary results of the clinical trial suggest the efficacy of the intervention, expecting that it may lead in a clinical and functional impact in this group of patients.

It can therefore be concluded that tasks presented in this work entail innovation in NPT's therapeutic contents from both a clinical and a technological perspective.

\section{ACKNOWLEDGMENT}

This research has been partially founded by the Spanish Ministry of Economy and Finance (project IPT-3000002010-30, NEUROCONTENT).

\section{REFERENCES}

1. World Health Organization (WHO) at http://www. who.int/

2. Couture S.M, Penn D.L, Roberts D.L. (2006) The Functional Significance of Social Cognition in Schizophrenia: A Review. Schizophrenia Bulletin 32:44-63 DOI 10.1093/schbul/sbl029

3. Green M.F, Olivier B, Crawley J.N et al. (2005) Social Cognition in Schizophrenia: Recommendations from the Measurement and Treatment Research to Improve Cognition in Schizophrenia. New Approaches Conference. Schizophrenia Bulletin 31:882-887 DOI $10.1093 / \mathrm{schbul} / \mathrm{sbi0} 49$

4. Krabbendam L, Aleman A. (2003) Cognitive rehabilitation in schizophrenia: a quantitative analysis of controlled studies. Psychopharmacology 169:376-382 DOI 10.1007/s00213-002-1326-5

5. Caballero-Hernández R, Martínez-Moreno J.M, García-Molina A et al. (2011) 2D-Tasks for Cognitive Rehabilitation. Proc. vol. 37, 5th European Conference of the International Federation for Medical and Biological Engineering (EMBEC 2011), Budapest, Hungary, 2011, pp 838-841

6. FranceschiniM, Agosti M, Cantagallo A, Sale P, Mancuso M, Buccino G. Mirror neurons: actions observation treatment as a tool in stroke rehabilitation. Eur J Phys Rehab Med 46 (2010), 517-523.

7. RehaCom at http://www.uam.es/personal_pdi/psicologia/pei/ pticexpo-0607/grupo8/software2.html

8. Gradior at http://www.intras.es/index.php/que-hacemos/idi/gradior

9. PssCogRegHab at http://www.neuroscience.cnter.com $/ \mathrm{pss} / \mathrm{psscr} . \mathrm{html}$

10. BrainTrain at $\mathrm{http} / / \mathrm{www}$.braintrain.com/

11. Solana J, García-Molina A et al. (2011) PREVIRNEC A new platform for cognitive tele-rehabilitation. The Third International Conference on Advanced Cognitive Technologies and Applications (COGNITIVE 2011), Roma, Italy; 2011. ISBN: 978-1-61208-155-7

12. Istitut Guttmann at http://www.guttmanninnova.com/

13. ParcTauli Mental Health Hospital at http://www.tauli.cat/

14. Orúe-Vega $\mathrm{O}$, Caballero-Hernández R,García-Molina A, MartínezMoreno JM,García-Rudolph A, Cáceres-Taladriz C,Tormos-Muñoz JM, Gómez-Aguilera EJ(2011) 'Entorno colaborativo de edición de tareas en neurorrehabilitación cognitiva', XXIX Congreso anual de la Sociedad Española de Ingeniería Biomédica, Cáceres. Libro de Actas CASEIB 2011, pp. 669-672.

15. J. M. Martínez Moreno, J. Solana Sánchez, R. Sánchez Carrión, S. González Palmero, P. Sánchez Gónzalez, C. Gómez Pérez, M. Morell Vilaseca, C. Cáceres Taladriz, T. Roig Rovira, J. M. Tormos Muñoz, E. J. Gómez Aguilera. Monitoring visual attention on a neurorehabilitation environment based on Interactive Video. International Conference on Recent Advances in Neurorehabilitation, Valencia. ICRAN Proceedings, 2013, pp. 182-185

Author: Ruth Caballero Hernández

Institute: Biomedical Engineering and Telemedicine Centre, ETSI Telecomunicación, Universidad Politécnica de Madrid

Street: Avda. Complutense 30

City: Madrid

Country: Spain

Email: rcaballero@gbt.tfo.upm.es 